\title{
Supplemental material of: Detecting and tracking eddies in oceanic flow fields: A vorticity based Euler-Lagrangian method
}

\author{
Rahel Vortmeyer-Kley ${ }^{1}$, Ulf Gräwe ${ }^{2,3}$, and Ulrike Feudel ${ }^{1}$ \\ ${ }^{1}$ Institute for Chemistry and Biology of the Marine Environment, Theoretical Physics/Complex Systems, Carl von Ossietzky \\ University Oldenburg, Oldenburg, Germany \\ ${ }^{2}$ Leibniz Institute for Baltic Sea Research, Rostock-Warnemünde, Germany \\ ${ }^{3}$ Institute of Meteorology and Climatology, Leibniz University Hannover, Hannover, Germany \\ Correspondence to: Rahel Vortmeyer-Kley (rahel.vortmeyer-kley@uni-oldenburg.de)
}

\section{S1 Seeded eddy model}

The model is inspired by the seeded eddy model of a turbulent flow described in Abraham (1998) and the model of Jung et al. (1993). The model consist of $n$ eddies and a background flow $u_{0}$ in positive $x$-direction.

The stream function for an eddy field with $n$ eddies is given by

$5 \quad \psi(\boldsymbol{x}, t)=\sum_{i=1}^{n}(-1)^{m_{i}} \cdot w_{i} \cdot \exp \left(-\kappa_{i}\left(\left(x-x_{i}\right)^{2}+\alpha\left(y-y_{i}\right)^{2}\right)\right)+u_{0} \cdot x$

where $\kappa_{i}^{-1 / 2}$ is linked to the radius of eddy $i$ with vortex strength $w_{i}$. The eddy $i$ emerges at position $\left(x_{i} y_{i}\right)$. The radii of the eddies are drawn from a distribution of 1000000 eddies possessing a power law $1 / r^{3}$. The distribution is normalized and allows eddies between $R_{\min }=10 \mathrm{~km}$ and $R_{\max }=50 \mathrm{~km}$. Because we are interested in mesoscale eddies $(15-25 \mathrm{~km}$ radii) we first draw a sample of 100 radii from the distribution and then pick randomly 10 sample radii that are between $15 \mathrm{~km}$ and $25 \mathrm{~km}$. The position of these eddies is drawn from an uniform distribution. Half of the eddies rotates counter-clockwise and the other half clockwise. The factor $m_{i}$ decides if the eddy rotates clockwise or counter-clockwise. If $m_{i}$ is even the eddy rotates clockwise if $m_{i}$ is odd the eddy rotates counter-clockwise.

The model is parametrized such that it yields a dimensionless model. Lengths are measured in units of the maximal eddy radius of the mesoscale eddies and time is in units of the lifetime of an eddy which is the same for all eddies. The parametrization is given in Table $\mathrm{S1}$.

\section{S2 The hydrodynamical model of a vortex street}

The hydrodynamical model of the vortex street is an adapted version of the model by Jung et al. (1993). Jung et al. (1993) models the flow in a channel behind a cylinder in the middle of the channel (cf. Jung et al. (1993) and Sandulescu et al. (2006) for details). 
Table S1. Dimensional and dimensionless parameters of the seeded eddy model

\begin{tabular}{ccccccc}
\hline Parameter & $r$ & $T_{c}$ & $\alpha$ & $\kappa$ & $u_{0}$ & $w$ \\
\hline dimensional & $25 \mathrm{~km}$ & $30 \mathrm{~d}$ & 1 & $1 / r^{2}$ & $0.18 \mathrm{~ms}^{-1}$ & $55 \cdot 10^{3} \mathrm{~m}^{2} \mathrm{~s}^{-1}$ \\
\hline dimensionless & 1 & 1 & 1 & 1 & 18.66 & 200 \\
\hline
\end{tabular}

Table S2. Dimensional and dimensionless parameters of the hydrodynamical model of a vortex street

\begin{tabular}{ccccccccc}
\hline Parameter & $r$ & $T_{c}$ & $\alpha$ & $\kappa$ & $L$ & $u_{0}$ & $y_{0}$ & $w$ \\
\hline dimensional & $25 \mathrm{~km}$ & $30 \mathrm{~d}$ & 1 & $1 / r^{2}$ & $150 \mathrm{~km}$ & $0.18 \mathrm{~ms}^{-1}$ & $12.5 \mathrm{~km}$ & $55 \cdot 10^{3} \mathrm{~m}^{2} \mathrm{~s}^{-1}$ \\
\hline dimensionless & 1 & 1 & 1 & 1 & 6 & 18.66 & 0.5 & 200 \\
\hline
\end{tabular}

Here this model is modified in such a way that two counter rotating eddies that develop at times $t$ and $t+T_{c} / 2$ at position $\left(1, y_{0}\right)$ and $\left(1,-y_{0}\right)$ respectively, travel a distance $L$ in positive $x$-direction within their lifetime $T_{c}$ and fade out. The model is artificial because the impact of the cylinder on the eddy formation and its shading are neglected here making the eddy formation non-physical out of the blue. However, since all quantities to be estimated by the eddy tracking tool are then given analytically,

5 makes this artificial model an ideal test bed for numerics.

Hence, the model is simplified as follows:

$\Psi(x, y, t)=-w h_{1}(t) g_{1}(x, y, t)+w h_{2}(t) g_{2}(x, y, t)+u_{0} y$

The first two terms describe the life cycle of the eddies of opposite sense of rotation. The vortex strength is given by $w$. The dynamics of the eddy evolution is modelled as the modulation of the amplitudes by the function $h_{1}(t)=\left|\sin \left(\pi t / T_{c}\right)\right|$ resp. $h_{2}(t)=h_{1}\left(t-T_{c} / 2\right)$. The function $g_{i}(x, y, t)=\exp \left(-\kappa_{0}\left(\left(x-x_{i}(t)\right)^{2}+\alpha\left(y-y_{i}(t)\right)^{2}\right)\right)$ with $i=1,2$ models the Gaussian shaped effect of the eddies on the stream function. The movement of the eddy centres is expressed by $x_{1}(t)=1+L\left(t / T_{c}\right.$ $\bmod 1), x_{2}(t)=x_{1}\left(t-T_{c} / 2\right)$ and $y_{1}(t)=y_{0}=-y_{2}(t)$. (In case of type 3 noise, the noise is added to the $y_{1}$ and $y_{2}$ terms.) The factor $\kappa_{0}^{-1 / 2}$ is the radius and determined as a characteristic linear size of the eddies and $\alpha$ is the ratio between the elongations of the eddy in $x$ and $y$ direction. In our case $\alpha$ is set to 1 (circular eddies). The last term describes the background flow with 15 the velocity $u_{0}$.

The parametrization of the flow is chosen as in Sandulescu et al. (2006). Lengths are measured in units of the eddy radius $r$ and time in units of the lifetime $T_{c}$ of an eddy. The dimensional and dimensionless parameters are given in Table $\mathrm{S} 2$. The vortex strength is furthermore varied to study its impact on the eddy evolution. 


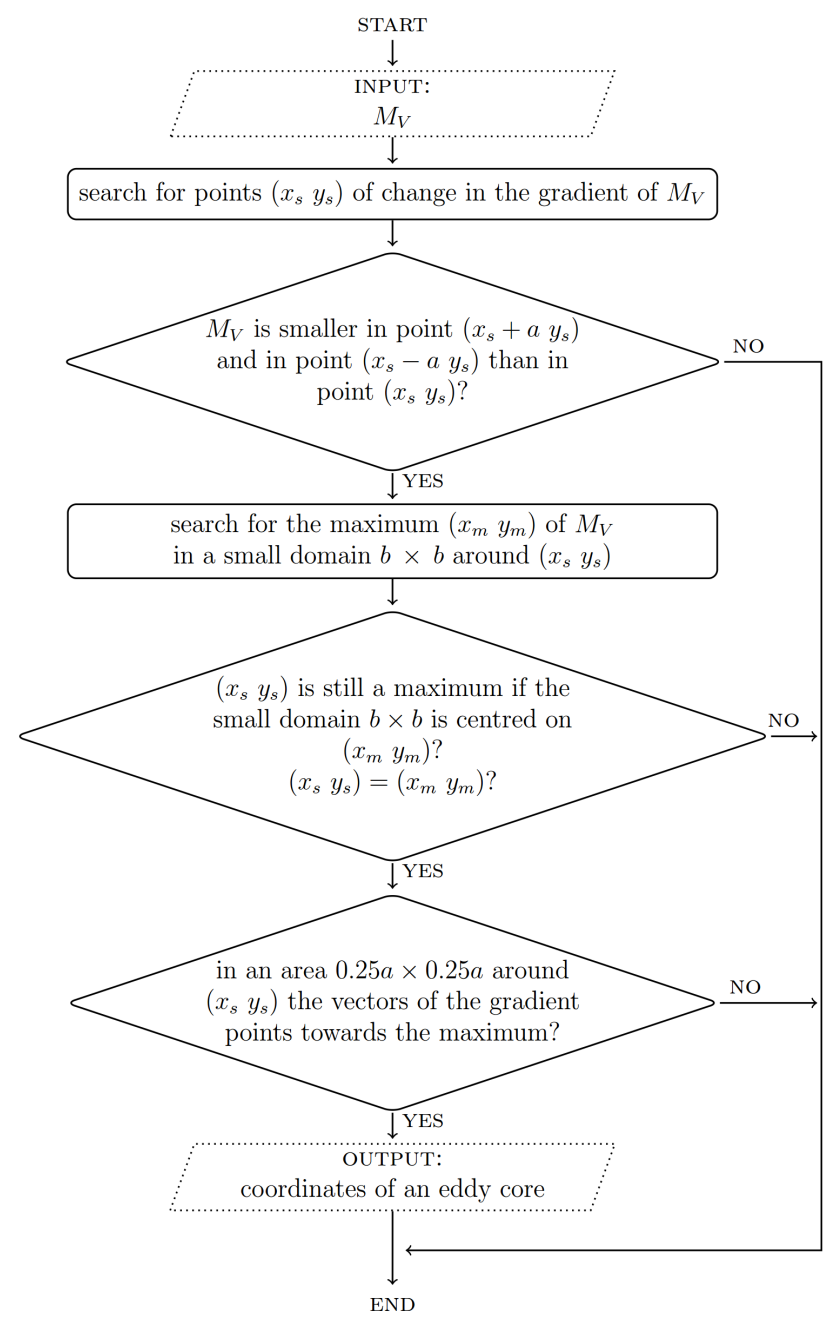

Figure S1. Schematic sketch of the eddy detection algorithm based on Euler-Lagrangian descriptor $M_{V}$.

\section{S3 Algorithm of the eddy tracking}

The key ideas of the algorithm (eddy detection, eddy tracking and eddy shape) inspired by the eddy tracking package by Nencioli et al. (2010) are schematically presented in this section.

Fig. S1] presents the concept of eddy detection. Similar algorithms can be constructed for the modulus of the vorticity and for 5 the Okubo-Weiss criterion (with respect to the fact that eddy cores are minima in case of Okubo-Weiss).

Fig. S2 shows idea of the eddy tracking. Fig. $\mathrm{S3}$ presents the eddy shape detection based on the combination of the EulerLagrangian descriptor $M_{V}$ and the Lagrangian descriptor $M$. 
INPUT VARIABLES EXPLANATION

$N$ cutoff number of time steps

(lower bound of the eddy lifetime)

$r$ the maximal distance a particle

can travel within one time step

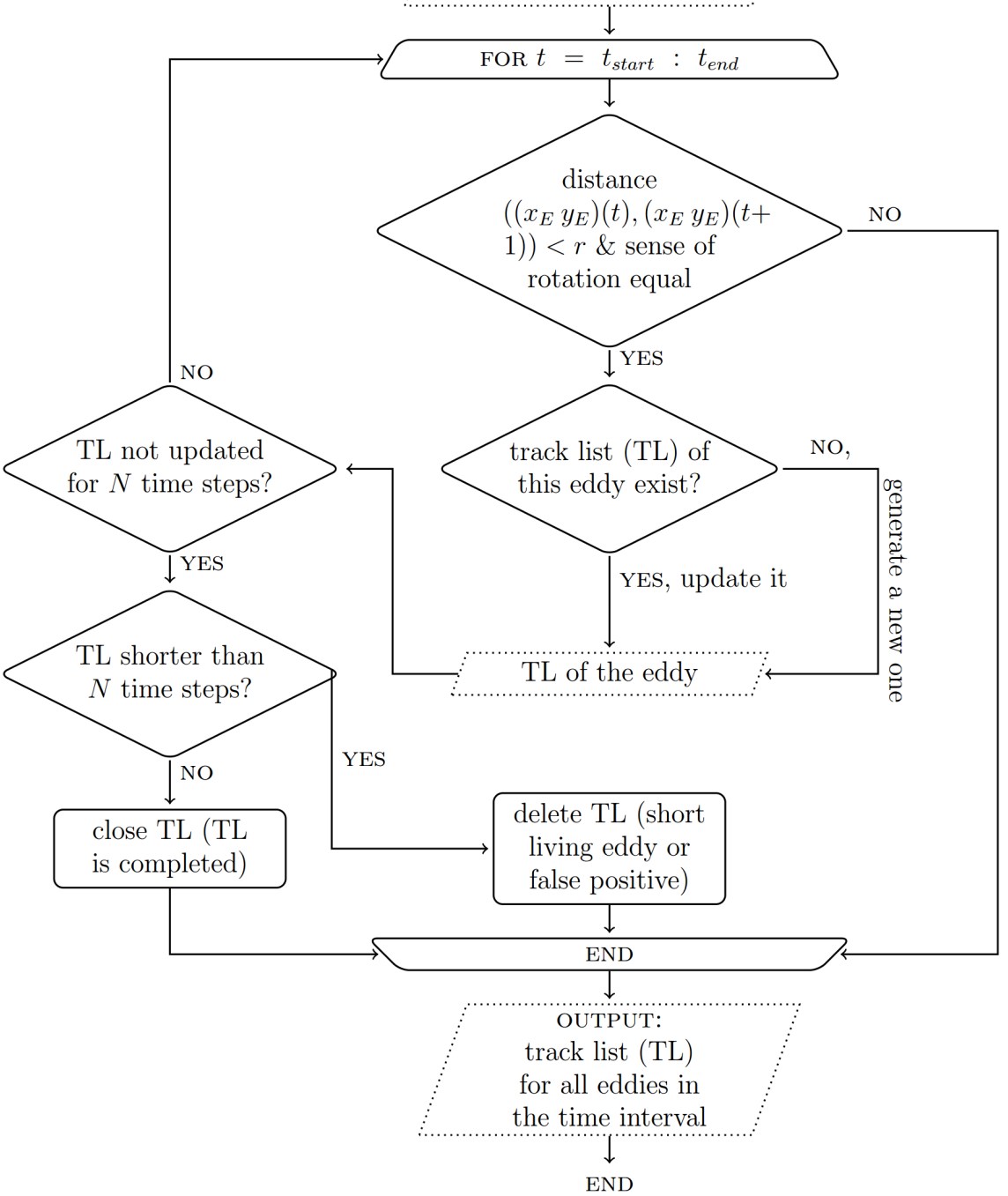

Figure S2. Schematic sketch of the eddy tracking algorithm based on Euler-Lagrangian descriptor $M_{V}$.

\section{References}

Abraham, E. R.: The generation of plankton patchiness by turbulent stirring, Nature, 391, 577-580, 1998.

Jung, C., Tél, T., and Ziemniak, E.: Application of scattering chaos to particle transport in a hydrodynamical flow, Chaos, 3, 555-568, 1993. 


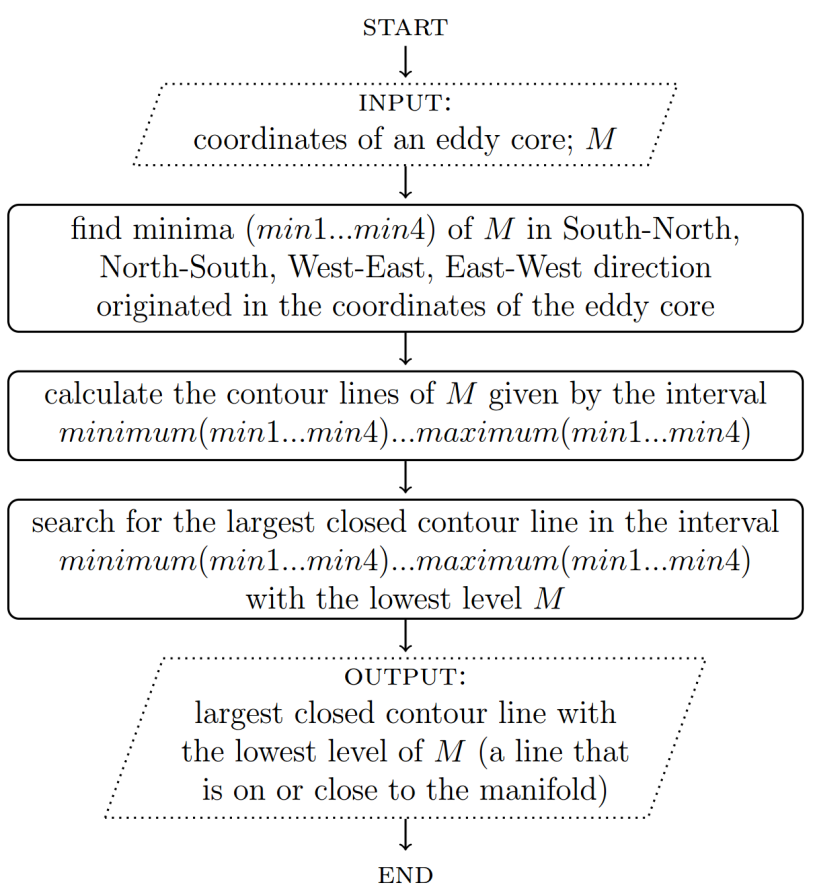

Figure S3. Schematic sketch of the eddy shape algorithm based on the combination of the Euler-Lagrangian descriptor $M_{V}$ and the Lagrangian descriptor $M$.

Nencioli, F., Dong, C., Dickey, T., Washburn, L., and McWilliams, J. C.: A Vector Geometry-Based Eddy Detection Algorithm and Its Application to a High-Resolution Numerical Model Product and High-Frequency Radar Surface Velocities in the Southern California Bight, J Atmos Ocean Tech, 27, 564-579, 2010.

Sandulescu, M., Hernández-García, E., López, C., and Feudel, U.: Kinematic studies of transport across an island wake, with application to Canary islands, Tellus A, 58, 605-615, 2006. 